\title{
Estrés post traumático e inadaptación en mujeres víctimas de violencia doméstica en la relación de pareja en función de la edad
}

Post-traumatic stress disorder (ptsd) and inadaptation in women victims by domestic violence in the marital relationship according to the age

Rosa E. Huerta R. ${ }^{1}$, Manuel Miljanovich C., Juan pequeña C., Emma Campos P., Renato Santivañez O., Jaime Aliaga T., Patricia Medina J., Pedro Pérez, Erika Fernández, Joel Uceda, Ana Silva, Robert Vidal

Universidad Nacional Mayor de San Marcos, Lima, Perù

(RECIBIDO 02-07 2014, ACEPTADO 20-10-2014)

\begin{abstract}
RESUMEN
La violencia doméstica es un grave problema social, por su alta frecuencia por la gravedad de los hechos y sus consecuencias negativas para las víctimas directas y personas cercanas.

En el estudio se sometió al análisis las variables; estrés postraumático e inadaptación en función a la edad, en una muestra de 256 mujeres víctimas de violencia doméstica en la relación de pareja, a quienes se les administró una ficha de datos sociodemográficos, la escala de gravedad de síntomas de estrés postraumático, la escala de inadaptación y una escala de síntomas somáticos de ansiedad. Se encontró una correlación significativa entre el estrés postraumático y la inadaptación en función a la edad en los diferentes grupos de edades. Así mismo, una correlación muy significativa entre cada uno de los componentes del estrés pos traumático, reexperimentación, evitación y activación con la inadaptación en todos los grupos de edad; apreciándose que las manifestaciones somáticas de la ansiedad en relación con la violencia doméstica también independientemente de la edad se encuentran relacionadas significativamente con la inadaptación. Por otro lado se encontró que la edad cronológica es una variable que no incide en los síntomas del estrés postraumático ni en el comportamiento de inadaptación, es la situación de violencia la que determina y produce los trastornos en la salud mental de la mujer, lo cual se observa en los valores medios obtenidos que resultan ser mayores que las medias de corte.
\end{abstract}

Palabras clave: Estrés post traumático, inadaptación, violencia doméstica, edad.

1 E-mail: huertarosel@hotmail.com 


\begin{abstract}
The domestic violence is a very big social problem, because of its high frequency and seriousness of the facts, and also its negative effects against the real victims and the close friends and relatives.

In the study, the variables of post-traumatic stress disorder and inadaptation were analyzed according to age in a sample of 256 female victims of domestic violence in the marital relationship, who were administered a sociodemographic data file, severity scale of posttraumatic stress disorder symptoms, the inadaptation scale and a scale of somatic symptoms of anxiety.

It was found a relevant correlation between the PTSD and inadaptation according to the age in the different age groups. In the same way, a very important correlation among each one of post-traumatic stress disorder components: reexperimentation, avoiding, and activation with the inadaptation in all kinds of age groups. It is observed that the somatic performance of anxiety, related to the domestic violence, apart from de age, has a significative relationship with the inadaptation. On the other hand, it was found that the chronological age is a variable that does not point out in the post-traumatic stress disorder symptom nor the inadaptation behavior. It is the violence situation that determines and produces the disorders in the woman's mental health, which is observed in the obtained mean values that result being more than the means of the cut stocking.
\end{abstract}

Keywords: Post-traumatic stress, inadaptation, domestic violence, age.

\title{
INTRODUCCIÓN
}

La violencia por parte de la pareja está asociada a una sensación de amenaza a la vida y al bienestar emocional por las graves implicaciones psicológicas que produce. Constituye un factor de riesgo para la salud mental, tanto a corto como a largo plazo, demostrado en numerosos estudios (Amor, Bohórquez, y Echeburúa, 2006; Soler, Barreto y González, 2005).

La violencia contra la mujer reviste diversas formas y representa un grave problema social. A su vez, los estereotipos sociales acerca del papel de la mujer y de las relaciones de pareja desempeñan un determinante en el mantenimiento de este tipo de violencia (Lorente: 2001). Dentro de este contexto, la violencia doméstica contra la mujer es un fenómeno epidémico que ha crecido a un ritmo más rápido, incluso que los accidentes de automóvil, las agresiones sexuales y los robos (Stark y Flitcraft, 1988). Este tipo de violencia no es reciente, pero actualmente se hace más visible. La sociedad ha tomado conciencia de su existencia y de que se trata de un grave problema social que no debe ocultarse por una mala entendida razón de familia, como algo privado.

En el Perú el Ministerio de la Mujer y Poblaciones Vulnerables ha registrado en el año 2012 un total de 26,960 casos de mujeres víctimas de violencia familiar entre 18 y 59 años de edad, en relación al total de casos que se atendieron, 42,537 de ambos géneros, apreciándose que son las mujeres las más maltratadas (reportes de MIMP, 2013; Endes 2012), repercutiendo negativamente en su salud mental. 
Miguel Ramos (citado por Guezmes, 2003) señala que la violencia contra las mujeres por parte de la pareja es un problema social generalizado, con altísimas prevalencias tanto en Lima Metropolitana como en el departamento de Cusco, las cuales se distribuyen de manera diferencial según edad. Son las mujeres más jóvenes las que sufren con mayor fuerza tales actos violatorios a sus derechos humanos.

Las investigaciones indican que las mujeres maltratadas experimentan enorme sufrimiento psicológico debido a la violencia. Una persona que viva con alguien que abusa de ella física o emocionalmente suele desarrollar una respuesta de estrés cuando es atacada. Si se repiten los ataques o amenazas, desarrolla una serie de síntomas crónicos, siendo los más prevalentes en las mujeres maltratadas por su pareja el trastorno de estrés postraumático y la depresión (Golding: 1999).

Se estima que el $60 \%$ de las mujeres maltratadas tienen problemas psicológicos moderados o graves (Lorente: 2001), los cuadros clínicos asociados más frecuentemente a la violencia doméstica son los trastornos de ansiedad, depresión, abuso y dependencia del alcohol y otras sustancias y, especialmente, el trastorno por estrés postraumático (Astin, Ogland-Hand, Foy y Coleman, 1995; Campbell, Sullivan y Davidson, 1995; Dutton, 1992; Golding, 1999; Labrador, Rincón, De Luis y Fernández-Velasco, 2004). El TEPT no solo es el trastorno de mayor prevalencia entre las víctimas de violencia doméstica, también es la categoría diagnóstica que mejor caracteriza las consecuencias psicológicas en estas personas, aunque puedan presentar síntomas de otros trastornos (Walker 2003).

La inadaptación a la vida cotidiana constituye una alarma en relación con la salud mental de la persona afectada. No apreciándose una clara distinción entre la normalidad y la anormalidad de los síntomas y de las conductas extrañas de una persona (Labrador, Echeburúa y Becoña, 2000).

Dominguez, García y Cuberos (2008), encontraron que los daños psicosociales como consecuencia de la violencia de pareja contra mujeres -usando la Escala de Inadaptación- que el $60 \%$ de ellas comunicaron que el maltrato les había generado problemas de salud, siendo los problemas psicológicos, depresión y ansiedad, los más frecuentes (32\%). Así mismo Huerta, R. et. al. (2012) encontraron que la presencia de ansiedad y depresión está presente en todos los tipos de convivencia de mujeres que viven en situación de violencia en la relación de pareja mostrando altos índices de ansiedad y depresión.

Otro estudio encontró que las víctimas de menor edad mostraban más síntomas depresivos y tenían una menor autoestima que las mayores. A su vez la gravedad del trastorno de estrés post traumático en las víctimas jóvenes estaba relacionado con la presencia de relaciones sexuales forzadas; en cambio en las víctimas adultas se relacionaba más con la percepción de amenaza contra sus vidas (Sarasua, Zubizarreta, Echeburúa y Corral; 2007). 


\section{Estrés post traumático}

El TEPT en las mujeres maltratadas suele estar acompañado de una desorganización completa del patrón de vida: abandono del hogar y la pareja, de los amigos, a veces del trabajo, de los lugares habituales y de referencia, tener que afrontar en solitario el cuidado y crianza de los hijos, problemas económicos, etc. Todo esto provoca, al menos en los primeros momentos, una grave inadaptación social y la pérdida de importantes redes de apoyo.

Según la versión revisada del DSM-IV (APA: 2000), cuya definición es similar de esta categoría diagnóstica respecto a la cuarta edición (APA: 1994), el TEPT aparece cuando la persona ha sufrido, ha sido testigo o le han explicado uno o más acontecimientos, caracterizados por muertes o amenazas para su integridad física o la de los demás, y cuando la reacción emocional experimentada implica una respuesta intensa de miedo, horror o indefensión.

Hay tres aspectos básicos de este cuadro clínico: la reexperimentación del suceso traumático en forma de pesadillas, imágenes y recuerdos constantes e involuntarios, la evitación conductual y cognitiva de los estímulos asociados al trauma y la hiperactivación fisiológica. Características que conllevan a una interferencia en el funcionamiento social, laboral y familiar, dificultades de concentración y conciliación del sueño, pérdida de interés por lo que antes le resultaba atractivo desde el punto de vista lúdico e interpersonal, y un cierto embotamiento afectivo para recibir y expresar sentimientos de intimidad y ternura.

El TEPT suele ser más grave y duradero cuando el suceso sufrido es muy intenso o cuando la víctima es vulnerable psicológicamente y no cuenta con un apoyo familiar y social adecuado (Resnick, Acierno, Amstadter, Self-Brown y Kilpatrick, 2007).

El TEPT, tiene una tasa media de prevalencia alta (entre un 45 y un $60 \%$ ) en la violencia de pareja (Cascardi et al., 1999; Amor et al., 2002 y Echeburúa et.al. 1997)

Estudios sobre la presencia de estrés post traumático manifiestan que suele ser un problema crónico y prolongado; las mujeres tardan alrededor de diez años de media en pedir ayuda, debido a las consecuencias personales, familiares y sociales en la mujer víctima de violencia doméstica (Arias et al., 1999).

\section{Violencia doméstica}

La violencia doméstica es definida como "un patrón de conductas abusivas, que incluye un amplio rango de maltrato físico, sexual y/o psicológico y que es ejercido por una persona en contra de otra, en el contexto de una relación íntima, con el objeto de ganar o mantener el abuso de poder, control y autoridad sobre ella" (Walker 1999: p.23).

Se consideran, principalmente, tres formas de violencia, que normalmente suelen aparecer interrelacionadas. La violencia física, violencia psicológica, y violencia sexual. Una cuarta forma que actualmente se incluye es la violencia económica la cual sucede con la intención de controlar el dinero de la mujer, coger su sueldo, no permitir el acceso al dinero familiar conllevando a que se incremente su temor y 
dependencia hacia la pareja y menoscabe sus posibilidades ocupacionales llegando inclusive a impedir que consiga o conserve un trabajo.

La permanencia de la mujer en la situación de violencia implica factores psicológicos y emocionales como los más importantes. Así, la autoculpabilización por la ocurrencia de las agresiones, el miedo a las represalias del agresor si le abandona, el temor a un futuro en soledad y la dependencia emocional de la víctima hacia él, favorecerían la permanencia de la víctima en la relación violenta. Además, las consecuencias emocionales que provoca el maltrato irían agotando los recursos psicológicos de estas mujeres para poder escapar de la situación.

Las consecuencias para las mujeres víctimas de maltrato son variadas, llegando en casos extremos a la muerte, ya sea por homicidio o por suicidio; en el Perú debido a esta grave consecuencia se ha incorporado el delito de feminicidio dentro del Código Penal Peruano mediante la Ley 29819 promulgada el 26 de diciembre del 2011.

Por otra parte, se estima que entre el 20 y $40 \%$ de las mujeres que se suicidan sufren malos tratos por parte de su cónyuge (Lorente: 2001). Los principales trastornos psicológicos desarrollados por este tipo de víctimas son la depresión, los trastornos de ansiedad y el trastorno de estrés postraumático (Montero, 2001; Walker, 1991). Muchas mujeres maltratadas no tienen conciencia de su victimización, y, en otros casos, no desean revelar que son víctimas de maltrato; así, tienden a minimizar, ocultar e incluso negar el maltrato del que son (o han sido) objeto.

\section{Inadaptación}

La inadaptación a la vida cotidiana constituye una alarma en relación con la salud mental de la persona afectada en los diferentes ámbitos en los que interacciona. La inadaptación es considerada como el desajuste en la salud mental, que produce un determinado trastorno en la vida cotidiana, refleja en qué medida los problemas psicológicos de los pacientes afectan a diferentes áreas de la vida cotidiana: trabajo o estudios, vida social, tiempo libre, relación de pareja y vida familiar.

Estudios de Labrador, et.al. (2004) señalan que un aspecto de especial relevancia es el alto nivel de inadaptación que se encuentran en las investigaciones en las mujeres maltratadas en su vida cotidiana. Ello es una alarma pues relieva de forma contundente los efectos desestructurantes que tiene la situación de maltrato en sus vidas. Las mujeres en esta situación ven afectada en primer lugar, su relación de pareja y su vida familiar. Después, mientras viven con el agresor, además del sufrimiento que la situación les genera, ven reducidas sus posibilidades de relacionarse con otras personas y de desarrollar actividades de ocio y tiempo libre debido a la situación de aislamiento y control que viven, llegando en algunos casos a una pérdida total de sus redes sociales. En tercer lugar, aquellas que ya han abandonado la situación de maltrato - que se encuentran viviendo en casas de familiares o amigos, en una casa de acogida o por su cuenta - se ven obligadas, en la mayoría de los casos y desde los primeros momentos, a realizar todas las labores domésticas y el cuidado de los hijos, a cambiar de trabajo, y a sumergirse en juicios o trámites legales, por lo que tampoco tienen posibilidades de recuperar o desarrollar su vida social ni actividades recreativas. 
Esta ruptura tan abrupta con toda su vida anterior y en condiciones generalmente adversas, hace muy difícil que puedan disponer de los recursos suficientes para lograr una nueva forma de inserción social. Las conductas que antes fueron adecuadas ahora no valen, las redes sociales que tenían se han difuminado, deben afrontar nuevas demandas sociales en condiciones de inestabilidad afectiva, personal y social, y además con frecuencia con dificultades económicas y miedo a la posible "venganza" del agresor por su denuncia. Las posibilidades de adaptación social son muy escasas.

Estudiar la inadaptación a la vida cotidiana ocupa un lugar central para examinar la existencia de muchos trastornos mentales conllevando a proponer la aplicación de un tratamiento efectivo para disminuirla, haciéndose su evaluación relevante para la intervención terapéutica pertinente y de gran apoyo en la práctica profesional y en la investigación clínica.

\section{Hipótesis}

H1: Existe una relación positiva entre el trastorno de estrés postraumático y la indaptación en mujeres víctimas de violencia doméstica en la relación de pareja en función a la edad, en la ciudad de Lima.

H2: Existen diferencias estadísticamente significativas en la presencia de estrés postraumático y la inadaptación en función a la edad en mujeres víctimas de violencia doméstica en la relación de pareja, de la ciudad de Lima.

H3: Existe relación entre el estrés postraumático y la inadaptación en mujeres víctimas de violencia doméstica.

\section{MÉTODO}

El presente estudio es de tipo sustantivo, que sistematiza procedimientos dentro del método correlacional y adicionalmente de la contrastación que nos permitió conocer tanto la intensidad y dirección de la relación entre el estrés portraumático y la inadaptación en mujeres con violencia en la relación de pareja según diferentes grupos de edad. El diseño fue descriptivo correlacional, transversal, los datos fueron recogidos en un solo momento (Hernández, Fernandez y Baptista, 2010).

\section{Muestra}

La muestra quedo constituida por 256 mujeres, que tienen o han tenido violencia en su relación de pareja y que asisten a los establecimientos de salud que atienden a mujeres con esta problemática de la ciudad de Lima. Aplicándose un muestreo no probabilístico, intencional; seleccionándose los establecimientos más representativos que atienden estos casos para su evaluación. La aplicación de los instrumentos se realizó de manera individual a través de una entrevista. La muestra se distribuyó en cuatro grupos de edades tal como se observa en la Tabla 1. 
Rosa E. Huerta R., Manuel Miljanovich C., Juan pequeña C., Emma Campos P., Renato Santivañez O., Jaime Aliaga T.

Tabla 1. Distribución de la muestra según grupo de edad

\begin{tabular}{cccc}
\hline Etapa de vida & Edad & f & \% \\
\hline Adolescentes & 16 a 21 años & 18 & 7,0 \\
Jóvenes & 22 a 35 años & 108 & 42,2 \\
Adulto joven & 36 a 45 años & 68 & 26,6 \\
Adulto & 46 a 65 años & 62 & $24, .2$ \\
$\mathbf{N}$ & & $\mathbf{2 5 6}$ & $\mathbf{1 0 0 , 0}$ \\
\hline
\end{tabular}

$\mathrm{Al}$ revisar en la muestra el tipo de convivencia al que pertenecen se observó que la familia nuclear presenta un porcentaje mayor $(42,2 \%)$ en toda la muestra, lo cual puede ser un indicador de permanencia en la relación de pareja a pesar de la existencia de violencia probablemente por su temor a la separación agravando el riesgo que implica ello; seguidas del tipo de familia extensa (20.5), monoparental (19.59) y la familia reconstituida (18.0). Así mismo al revisar el tipo de violencia, según la declaración de víctimas, se observa que la violencia psicológica ocupa porcentualmente el primer lugar seguido por la violencia física, ambas son relativamente frecuentes más aun la psicológica que llega al 97.7 por ciento y 70.7 por ciento; por otro lado, la violencia económica y sexual, porcentualmente tienen presencia de 23.0 y 17.3 por ciento respectivamente.

\section{Instrumentos}

Para la presente investigación se utilizaron los siguientes instrumentos, aplicados a través de la entrevista individual, las mujeres fueron examinadas en los establecimientos donde se atienden mujeres víctimas de violencia en la relación de pareja. Las escalas que a continuación se describen fueron previamente adaptadas lingüísticamente.

Ficha de datos sociodemográficos: Elaborada por el investigador, recoge información del lugar de procedencia, edad, grado de instrucción, número de compromisos, el tipo de convivencia, tiempo de convivencia así como los tipos de violencia en la que se encuentran involucradas.

Escala de gravedad de síntomas del trastorno de estrés postraumático (Echeburúa, Corral, Amor, Zubizarreta y Sarasua, 1997b). Es una entrevista estructurada que evalúa la gravedad e intensidad de los síntomas de este cuadro clínico -según los criterios diagnósticos del DSM-IV (American Psychiatric Association, 1994). La escala está estructurada en un formato de tipo Likert de 0 a 3 según la frecuencia e intensidad de los síntomas, consta de 17 ítems, de los cuales 5 se refieren a los síntomas de reexperimentación, 7 a los de evitación y 5 a los de hiperactivación. El rango es de 0 a 51 en la escala global, de 0 a 15 en la subescala de reexperimentación, de 0 a 21 en la de evitación y de 0 a 15 en la de activación. La eficacia diagnóstica de la escala es muy alta $(95,45 \%)$, con un punto de corte global de 15 y puntos de corte parciales de 5, 6 y 4 en las subescalas de reexperi- 
mentación, evitación e hiperactivación, respectivamente. Para la validez se realizó el método de consistencia interna para lo cual se ha correlacionado cada subtest utilizando el coeficiente de Correlación Rho de Spearman, encontrando que cada una de las subescalas, presentan correlaciones significativas, inter subescalar y subescala, test total, indicando que las mencionadas subescalas están asociados y miden un mismo factor. La confiabilidad se realizó utilizando el procedimiento alfa de Cronbach, para cada sub escala y luego para el test total, encontrando 0.804 para reexperimentación, 0.79 para evitación, 0.77 para hiperactivación y, para la escala global 0.899. Por lo que podemos afirmar que la escala es confiable, los niveles obtenidos son similares a los de sus autores (Echeburúa et al., 1997b).

Escala de inadaptación (Echeburúa y Corral, 1995). Es un autoinforme que consta de 6 ítem (rango: 0-30 puntos) y evalúa el grado en que el maltrato doméstico afecta a diferentes áreas de la vida cotidiana: trabajo, vida social, tiempo libre, relación de pareja y relación familiar. El punto de corte es de 12 en la escala total y de 2 en cada uno de los ítems. Cuanto mayor es la puntuación, mayor es la inadaptación. En esta investigación se ha utilizado el ítem que refleja el grado de inadaptación global a la vida cotidiana, con un rango que oscila de 0 a 5 puntos. Para la validez, se utilizó la validez concurrente bajo el supuesto que esta variable va asociada a los síntomas del trastorno del estrés postraumático, encontrando coeficientes significativos. Revisando la confiabilidad de la Escala de Inadaptación se encontró un coeficiente de 0.917 , por tanto se recomienda su uso para el estudio de esta variable. Niveles altos similares a lo observado por sus autores (Echeburúa, Corral y Fernández-Montalvo (2000).

Escala complementaria de síntomas somáticos de ansiedad. Se utilizó esta escala como confirmatoria de los indicadores de ansiedad. Son los criterios planteados en el DSM IV-TR para los Trastornos de ansiedad y crisis de pánico; permite observar 13 síntomas de ansiedad, categorizadas en un rango de 0-39 para la escala total y de 0 a 3 para cada ítem. Según el DSMIV TR se requiere de 4 síntomas o más y como punto de corte para la escala total se han considera 12 puntos. Tanto sus índices de validez como confiabilidad son significativos, llegando hasta niveles de 0.58 .

\section{RESULTADOS}

Relación entre el trastorno postraumático y la inadaptación en mujeres víctimas de violencia doméstica en función a la edad. Para conocer la correlación entre los síntomas del trastorno de estrés postraumático con la inadaptación se utilizó el coeficiente de correlación (Rho) de Spearman para cada subescala y según el grupo por edad y prescindiendo de ella y se ha encontrado:

a. La reexperimentación y la Inadaptación se correlacionan significativamente en todos los grupos de edad sean estas adolescentes, adultas jóvenes, adultas o adultas mayores y se confirma con el coeficiente hallado teniendo en cuenta todas las edades, hecho que nos dice que la edad no atenúa ni acentúa más el estrés postraumático y el 
comportamiento adaptativo sino que ambos interaccionan provocando inestabilidad emocional e interferencias en los diferentes ámbitos en que se desenvuelve, social, familiar, laboral y pérdida del interés en las actividades que antes realizaba.

b. En la evitación con la inadaptación los coeficientes son mayores aun en todas las edades, cuando aumenta los síntomas del trastorno del estrés postraumático aumenta el comportamiento de inadaptación por lo que no quieren aceptar ni tocar el tema, tendiendo a minimizar la situación de violencia vivida y agravando el aislamiento de su familia y amigos. Así, frente a las razones del porque no lo denunció señalan "es simple", "pensé que era normal”, "no creo ser víctima de violencia”, entre otros.

c. El aumento de la activación con inadaptación se correlacionan significativamente en todas las edades, por lo que cuando aumenta una de ellas también aumentará la otra pues la mujer víctima de violencia se encuentra en estado permanente de temor de que vuelva a ocurrir el hecho de violencia, causándole respuestas inadaptativas como un estado de ánimo irritable, problemas en su salud física y desesperanza.

d. Las manifestaciones somáticas de la ansiedad en relación con la violencia domésticas también independientemente de la edad se encuentra relacionada significativamente con la inadaptación, es decir hay una presencia significativa de ansiedad asociada que van a incrementar su desapego por los demás, restringiendo su vida afectiva y social.

Tabla 2. De coeficientes de correlación (Rho) entre las subescalas y escalas de estrés postraumático e inadaptación en mujeres víctimas de violencia doméstica

\begin{tabular}{|c|c|c|c|c|c|c|c|}
\hline & & & $\begin{array}{c}\text { T_EI } \\
16 \text { a } 21\end{array}$ & $\begin{array}{c}\text { T_EI } \\
22 \text { a } 35\end{array}$ & $\begin{array}{c}\text { T_EI } \\
36 \text { a } 45\end{array}$ & $\begin{array}{c}\text { T_EI } \\
46 \text { a } 65\end{array}$ & $\begin{array}{l}\text { T_EI Todas } \\
\text { las edades }\end{array}$ \\
\hline \multirow[t]{15}{*}{$\begin{array}{c}\text { Rho de } \\
\text { Spearman }\end{array}$} & T_Gre & $\begin{array}{l}\text { Coeficiente de } \\
\text { correlación }\end{array}$ & .441 & $.551(* *)$ & $.602(* *)$ & $.562(* *)$ & $.554(* *)$ \\
\hline & & Sig. (bilateral) & .067 & .000 & .000 & .000 & .000 \\
\hline & & $\mathrm{N}$ & 18 & 108 & 76 & 54 & 256 \\
\hline & T_Gevi & $\begin{array}{l}\text { Coeficiente de } \\
\text { correlación }\end{array}$ & $.575(*)$ & $.685(* *)$ & $.759(* *)$ & $.684(* *)$ & $.705(* *)$ \\
\hline & & Sig. (bilateral) & .012 & .000 & .000 & .000 & .000 \\
\hline & & $\mathrm{N}$ & 18 & 108 & 76 & 54 & 256 \\
\hline & T_Aac & $\begin{array}{l}\text { Coeficiente de } \\
\text { correlación }\end{array}$ & $.574(*)$ & $.655(* *)$ & $.596(* *)$ & $.605(* *)$ & $.619(* *)$ \\
\hline & & Sig. (bilateral) & .013 & .000 & .000 & .000 & .000 \\
\hline & & $\mathrm{N}$ & 18 & 108 & 76 & 54 & 256 \\
\hline & T_Eglobal & $\begin{array}{l}\text { Coeficiente de } \\
\text { correlación }\end{array}$ & $.662(* *)$ & $.704(* *)$ & $.770(* *)$ & $.696(* *)$ & $.718(* *)$ \\
\hline & & Sig. (bilateral) & .003 & .000 & .000 & .000 & .000 \\
\hline & & $\mathrm{N}$ & 18 & 108 & 76 & 54 & 256 \\
\hline & T_Ecomp & $\begin{array}{l}\text { Coeficiente de } \\
\text { correlación }\end{array}$ & .049 & $.522(* *)$ & $.586(* *)$ & $.282(*)$ & $.473(* *)$ \\
\hline & & Sig. (bilateral) & .848 & .000 & .000 & .039 & .000 \\
\hline & & $\mathrm{N}$ & 18 & 108 & 76 & 54 & 256 \\
\hline
\end{tabular}

* La correlación es significativa al nivel 0,05 (bilateral).

** La correlación es significativa al nivel 0,01 (bilateral). 
Para verificar si efectivamente la edad es independiente a las correlaciones encontradas entre los síntomas de trastorno del estrés postraumático con la conducta inadaptativa se correlacionó la edad con cada una de las escalas y como se puede observar en la siguiente tabla, todas las correlaciones no son significativas, confirmando que la edad es una variable independiente a la sintomatología y a la inadaptación, no siendo la edad la que genera la violencia sino la presencia de violencia en la relación de pareja, en cualquier etapa de la vida la que genera problemas en la salud mental de la mujer víctima, tal como se puede ver a continuación

Tabla 3. De coeficiente de correlación entre la edad con cada escala, totales de los síntomas del trastorno del estrés postraumático y la escala de inadaptación

\begin{tabular}{|c|c|c|c|c|c|c|c|c|c|}
\hline & & & Edad & T Gre & T Gevi & T Aac & T Eglobal & T Ecomp & T EI \\
\hline \multirow[t]{3}{*}{$\begin{array}{c}\text { Rho de } \\
\text { Spearman }\end{array}$} & Edad & $\begin{array}{c}\text { Coeficiente de } \\
\text { correlación }\end{array}$ & 1.000 & -.029 & .017 & -.023 & -.025 & 109 & .087 \\
\hline & & Sig. (bilateral) & . & .643 & .787 & .713 & .690 & .081 & .166 \\
\hline & & $\mathrm{N}$ & 256 & 256 & 256 & 256 & 256 & 256 & 256 \\
\hline
\end{tabular}

Diferencia del trastorno del estrés post traumático y la inadaptación en mujeres víctimas de violencia doméstica en función a la edad - para conocer si la edad plantea diferencias significativas en cada escala de síntomas de trastorno de estrés postraumático y la inadaptación hemos contrastado los valores registrados de las mujeres víctima de violencia doméstica y contrastado según la edad, agrupando en adolescentes entre 16 a 21 años, adultos jóvenes de 22 a 35, adultos de 36 a 45 años, adultos mayores entre los 46 a 65 años de edad, se ha encontrado, confirmando lo hallado en el análisis correlacional, que la edad cronológica es una variable que no incide en los síntomas del trastorno de estrés postraumático ni en el comportamiento de Inadaptación, la violencia marca y trastorna, se puede observar por los valores medios obtenidos que resultan ser mayores que las medias de corte consideras por los autores, como se puede ver en la siguiente tabla:

Tabla 4. Resumen de Análisis de varianza de los puntajes por cada subescala y comportamiento Inadaptación según la edad

\begin{tabular}{lccccc}
\cline { 2 - 5 } & $\mathrm{F}$ & $\mathrm{P}$ & $\mathrm{F}$, & $\begin{array}{c}\text { Media } \\
\text { Obtenida }\end{array}$ & $\begin{array}{c}\text { Media de } \\
\text { Corte }\end{array}$ \\
\hline Rexperimentación & 0.5974 & 0.617 & 2.64 & 8.08 & 5 \\
Evitación & 0.0987 & 0.9606 & 2.64 & 11.08 & 6 \\
Aumento Activación & 0.434 & 0.7291 & 2.64 & 8.617 & 4 \\
Global & 0.3515 & 0.8025 & 2.64 & 27.79 & 15 \\
Escala Complementaria & 0.4879 & 0.69 & 2.64 & 12.62 & 12 \\
Inadaptación & 0.6884 & 0.5598 & 2.64 & 18.95 & 12 \\
\hline
\end{tabular}

Relación entre el trastorno postraumático y la inadaptación en mujeres víctimas de violencia doméstica - ambas variables tienen un intensidad de correlación de 0.718 que resulta muy significativa aun para el 0.0001 de margen de error, por lo que se puede afirmar que los síntomas del trastorno postraumático del estrés resulta 
interdependiente con la inadaptación, que nos dice que la salud mental de la mujer víctima de violencia doméstica se ve agravada no solamente por la sintomatología del estrés postraumático de permanente temor, desesperanza, aislamiento, desapego de los demás sino que además ello trae como consecuencia en su vida cotidiana, obstaculizando y limitando su vida afectiva, familiar, social y laboral, tal como se puede ver en la siguiente tabla:

Tabla 5. De coeficientes de correlación entre los síntomas del estrés postraumático global y escala complementaria de síntomas de ansiedad con la inadaptación en mujeres víctimas de violencia doméstica

\begin{tabular}{cclc}
\cline { 2 - 3 } Rho de Spearman & & T_EI Todas las edades \\
& T_Eglobal & Coeficiente de correlación & $.718\left(^{* *}\right)$ \\
& & Sig. (bilateral) & .000 \\
& $\mathrm{~N}$ & 256 \\
& \multirow{2}{*}{ T_Ecomp } & Coeficiente de correlación & $.473\left(^{* *}\right)$ \\
& Sig. (bilateral) & .000 \\
& $\mathrm{~N}$ & 256 \\
\hline
\end{tabular}

** La correlación es significativa al nivel 0,01 (bilateral).

En el gráfico 1, se observa que los puntajes obtenidos en la Escala de Gravedad de Síntomas del Estrés Postraumático a nivel global, superan los puntajes de corte de la prueba, apreciándose que son las mujeres más jóvenes (adolescentes) las que presentan un ligero mayor puntaje, y que en los otros grupos de edades este es alto.

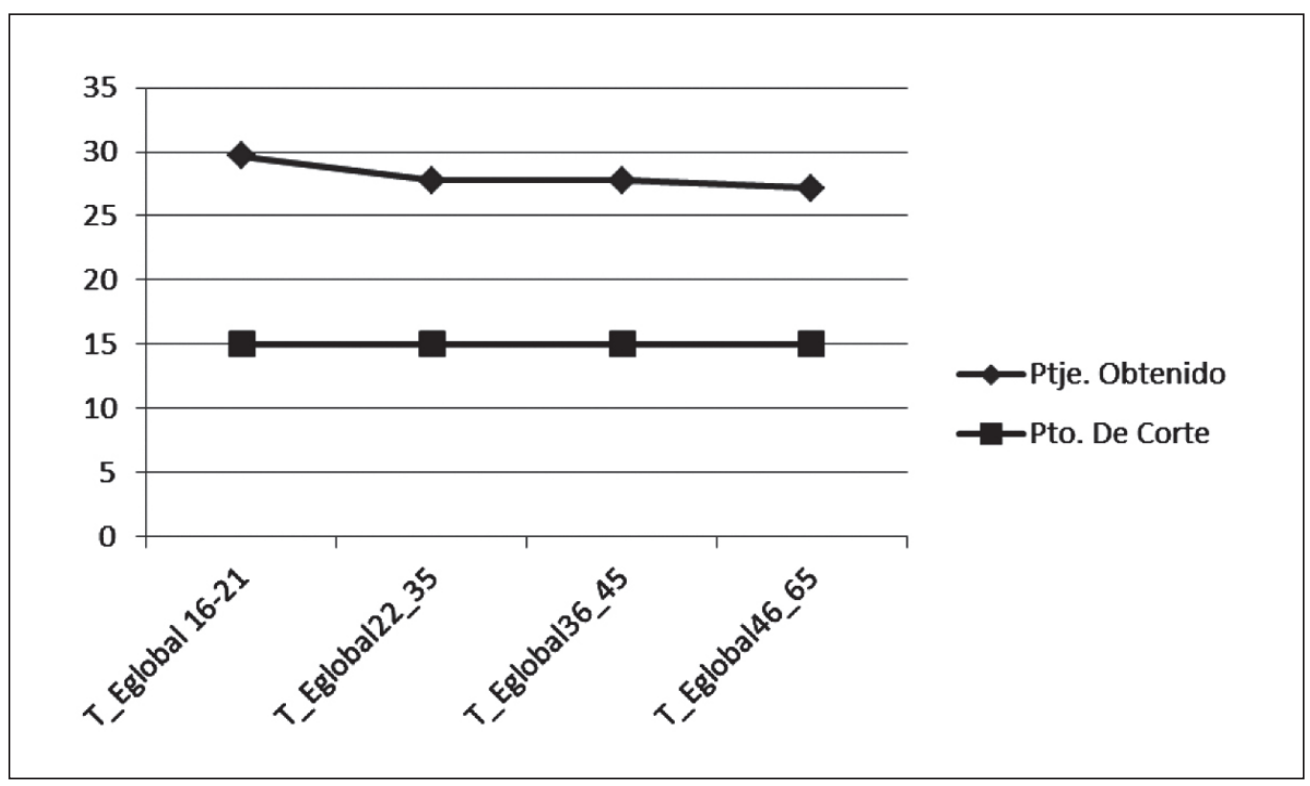

Grafico $\mathrm{N}^{\circ}$ 1. Perfil de la escala global de los síntomas de trastorno de estrés postraumático en mujeres víctimas de violencia doméstica 
- Análisis de la escala de inadaptación por cada uno de las aspectos y en general según la edad en mujeres víctimas de violencia doméstica.- para este análisis los resultados obtenidos de los seis aspectos que explora la escala de Inadaptación, para cada periodo cronológico se calculó las medias aritméticas con el objeto de ubicar en qué valor de inadaptación se encuentran y luego se contrastó, utilizando el análisis de varianza encontrando que la edad cronológica no es un factor de incidencia, ya que como se podrá leer en la siguiente tabla no se ha registrado ninguna razón F significativa: sobre estos datos obsérvese que en todos los casos las medias aritméticas son mayores de 2.5 que es señal de la presencia de la violencia doméstica independientemente de las edades aparta a la víctima de la realidad, apoyándose como principal causa el maltrato disminuyendo la posibilidad de enfrentamiento hacia una resignación progresiva que conllevan temor, desesperanza e indefensión, tal como se puede ver en la siguiente tabla y gráfico.

Tabla 6. Resumen descriptiva y de análisis de varianza de los aspectos de la Escala de Inadaptación en mujeres víctimas de violencia doméstica

\begin{tabular}{lccccccc}
\hline Aspectos de la E.I & 16 a 21 & 22 a 35 & 36 a 45 & 46 a 65 & $F$ & P & $F^{\prime}$ \\
\hline Trabajo y/o estudio & 3.33 & 2.92 & 2.99 & 3.06 & 0.64 & 0.59 & 2.64 \\
Vida social & 3.11 & 2.80 & 3.07 & 3.07 & 1.06 & 0.67 & 2.64 \\
Tiempo libre & 3.17 & 3.00 & 3.01 & 3.04 & 0.09 & 0.94 & 2.64 \\
Relación de pareja & 3.39 & 3.27 & 3.37 & 3.35 & 0.1 & 0.95 & 2.64 \\
Vida familiar & 3.44 & 3.07 & 3.32 & 3.41 & 1.18 & 0.32 & 2.64 \\
Escala global & 3.50 & 3.25 & 3.57 & 3.48 & 1.19 & 0.31 & 2.64 \\
Total general & 3.32 & 3.32 & 3.32 & 3.32 & 0.68 & 0.56 & 2.64 \\
\hline
\end{tabular}

Tal como se puede visualizar en el gráfico

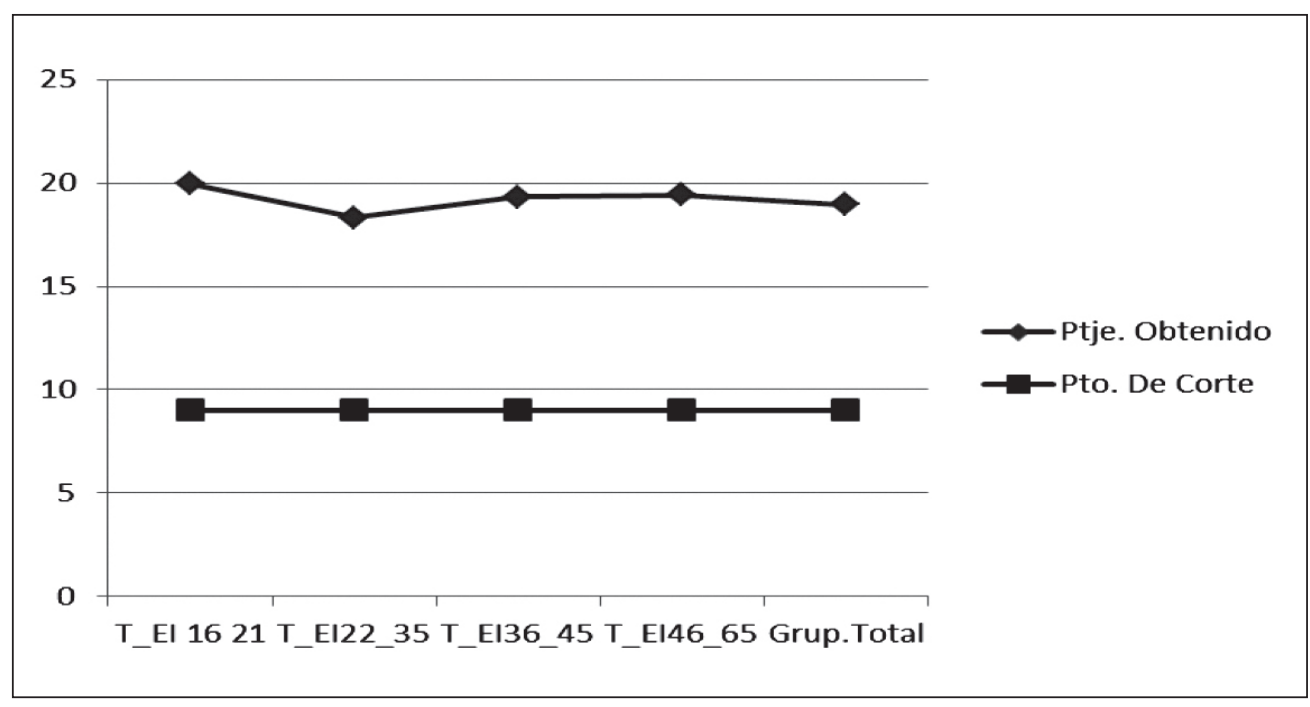

Grafico $\mathrm{N}^{\circ}$ 2. Perfil de la escala de inadaptación en mujeres víctimas de violencia doméstica 
Análisis complementario - con los datos obtenidos es necesario localizar los valores descriptivos en las variables estudiadas, con el propósito de localizar la intensidad de los trastornos postraumáticos del estrés y de la Inadaptación; para este fin se ha calculado las medidas de tendencia central primero por grupo de edad y luego para la muestra total sin considerar la edad, a pesar que ya se indicó que no hay diferencias según la edad, apreciándose que todas superan los puntos de corte de los instrumentos, planteados por los autores; la presentación grafica adicional permite visualizar lo hallado:

Tabla 7. De Medidas y Desviaciones estándar por subescala y total de Síntomas de Estrés

Postraumático y de Inadaptación en mujeres víctimas de violencia domésticas según la edad

\begin{tabular}{|c|c|c|c|c|c|c|c|c|c|}
\hline & Periodo & corte & Media & DS & & Periodo & corte & Media & D.S \\
\hline \multirow[t]{5}{*}{ Rexperimentación } & Adolescente & 16 a 21 & 8.94 & 3.11 & Global & Adolescente & 16 a 21 & 29.67 & 8.46 \\
\hline & Jóvenes & 22 a 35 & 8.16 & 3.32 & & Jóvenes & 22 a356 & 27.81 & 9.65 \\
\hline & Adulto Jov. & 36 a 45 & 7.91 & 2.66 & & Adulto Jov. & $36 \mathrm{~s} 45$ & 27.78 & 8.58 \\
\hline & Adulto & 46 a 65 & 7.93 & 3.36 & & Adulto & 46 a 65 & 27.17 & 9.40 \\
\hline & Grupo total & & 8.09 & 3.13 & & Grupo total & & 27.79 & 9.18 \\
\hline \multirow[t]{5}{*}{ Evitacion } & Adolescente & 16 a 21 & 11.56 & 3.57 & Complementos & Adolescente & 16 a 21 & 11.89 & 3.32 \\
\hline & Jóvenes & 22 a35 & 11.06 & 4.19 & & Jóvenes & 22 a 356 & 12.32 & 5.35 \\
\hline & Adultos & $36 \mathrm{~s} 45$ & 11.11 & 4.12 & & Adultos & $36 \mathrm{~s} 45$ & 12.88 & 5.17 \\
\hline & Adulto mayor & 46 a 65 & 10.94 & 4.33 & & Adulto mayor & 46 a 65 & 13.09 & 4.34 \\
\hline & Grup total & & 11.09 & 4.14 & & Grupo total & & 12.62 & 4.97 \\
\hline \multirow[t]{5}{*}{ A. de la activación } & Adolescente & 16 a 21 & 9.17 & 2.92 & C. Inadaptación & Adolescente & 16 a 21 & 19.94 & 5.29 \\
\hline & Jóvenes & 22 a35 & 8.58 & 3.43 & & Jóvenes & 22 a 356 & 18.31 & 6.10 \\
\hline & Adultos Jov. & $36 \mathrm{~s} 45$ & 8.76 & 3.00 & & Adulto Jov. & $36 \mathrm{~s} 45$ & 19.32 & 7.10 \\
\hline & Adulto $\mathrm{r}$ & 46 a 65 & 8.30 & 2.72 & & Adulto & 46 a 65 & 19.41 & 6.18 \\
\hline & Grupo total & & 8.62 & 3.12 & & Grup total & & 18.95 & 6.37 \\
\hline
\end{tabular}

\section{DISCUSIÓN}

La violencia doméstica hacia la mujer en la relación de pareja reviste graves consecuencias para su salud mental. Los estudios sobre estrés postraumático coinciden en asociarla a este tipo de violencia, es así que Walker (2003) señala que el trastorno de estrés postraumático es el trastorno de mayor prevalencia entre las víctimas de violencia doméstica y que además es la categoría diagnóstica que 
mejor caracteriza las consecuencias psicológicas en estas personas; también se asocian a otros trastornos como son la ansiedad, depresión, abuso y dependencia al alcohol, especialmente el estrés postraumático (Astin, Ogland-Hand, Foy y Coleman, 1995; Campbell, Sullivan y Davidson, 1995; Dutton, 1992; Golding, 1999; Labrador, Rincón, De Luis y Fernández-Velasco, 2004). Datos similares encontramos en el presente estudio pues todas las mujeres, en los diferentes grupos de edades presentaron altos indicadores de TEPT, alcanzando para la muestra total en la Escala Global 27.79 de media, dato muy superior al punto de corte, propuesto por los autores que es 15. Lo cual implica una respuesta de estrés cuando es atacada, desarrollando así un problema crónico y prolongado, un estado de permanente vigilancia, vivencia de fracaso personal, desesperanza y pérdida de la autoestima así como una grave inadaptación social y pérdida de sus redes de apoyo familiares, amicales y laborales.

En lo referente al grupo etareo y TEPT, Sarasúa, et. al. (2007), en un estudio con mujeres víctimas de violencia en la relación de pareja encontró que la tasa de prevalencia del TEPT era más alta en las víctimas más jóvenes de 18 a 29 años (42\%) que en las mayores de 30 a 69 años (27\%). En el presente estudio todos los grupos de edades presentan síntomas de TEPT y, coincide con los resultados de Sarasua, et. al., es decir son las más jóvenes (16 a 21 años) quienes alcanzan una media relativamente más alta (29.67) en relación con los otros grupos de edades 22 a 35 años (27.81), 36 a 45 años (27.78) y 46 a 65 años (27.17) lo que hace más grave la permanencia en la relación de pareja y de mayor riesgo.

Por otro lado, en lo referente a la prevalencia de maltrato hacia la mujer como lo señala el estudio multicéntrico de la OMS sobre la violencia de pareja y las salud de las mujeres (Guezmes, et, al. 2002), existe una alta prevalencia en Lima Metropolitana (51\%), con preponderancia en las mujeres más jóvenes (22 a 29 años). Similar resultado encontramos en la muestra estudiada, son las mujeres jóvenes (22 a 35 años) las que presentan mayor porcentaje de violencia en la relación de pareja $(42.2 \%)$.

Cuando se revisa el tipo de violencia predominante en la mujer maltratada, tanto los estudios del MIMP (2013), como el de Endes 2012, señalan al maltrato psicológico como el tipo de violencia más predominante. Alcanzando el maltrato psicológico según Endes 2012 un $66.3 \%$, ya sea en la forma de control, amenaza o humillación. Dato similar encontramos en el presente estudio, es decir las mujeres reportaron mayor violencia psicológica, $97.7 \%$ seguido de la violencia física, económica y sexual, es relevante considerar las consecuencias en la salud mental de las mujeres víctimas, como plantea Sarasua, et.al (2007), la mujer tiende a recordar más el primer acto de violencia física y no la psicológica confundiéndola, lo que hace que el trauma se vuelva crónico e interfiera en su vida cotidiana. La permanencia de la mujer en la situación de violencia implica factores psicológicos y emocionales. Así, se a autoculpabiliza por la ocurrencia de las agresiones, teme a las represalias del agresor si le abandona, teme un futuro en soledad que se agrava por la dependencia emocional de la víctima hacia él, lo que favorecería 
la permanencia de la víctima en la relación violenta. Además, las consecuencias emocionales que provoca el maltrato irían agotando los recursos psicológicos de estas mujeres para poder escapar de la situación.

La intensidad de los síntomas del TEPT, inmediatamente tras la agresión es un predictor del funcionamiento de las víctimas a largo plazo (Resnick, Acierno, Amstadter, Self-Brown y Kilpatrick, 2007). Ello es alarmante pues la edad cronológica no incide en la presencia de síntomas de estrés postraumático ni en la inadaptación a la vida cotidiana es la situación de violencia la que interfiere y anula las posibilidades cognitivas, emocionales, afectivas y sociales de la víctima como se puede observar en todas las áreas de la inadaptación (trabajo y estudio, vida social, tiempo libre, vida familiar y especialmente en la relación de pareja) y en todos los grupos de edades, no encontrándose ninguna razón $\mathrm{F}$ significativa pero si una media mayor a 2.5, lo que es indicador que la presencia de violencia doméstica independiente de las edades aparta a la víctima de su realidad, interfiriendo en su vida cotidiana, lo cual se visualiza en el puntaje alto alcanzado en la escala global de inadaptación (18.95), siendo el punto de corte 12. Estudios de Labrador, et.al. (2004) señalan que un aspecto de especial relevancia es el alto nivel de inadaptación que se encuentran en las investigaciones las mujeres maltratadas en su vida cotidiana. Ello es una alarma pues relieva de forma contundente los efectos desestructurantes que tiene la situación de maltrato en sus vidas. Las mujeres en esta situación ven afectada, en primer lugar, su relación de pareja y su vida familiar. Datos que reafirman en sus investigaciones Amor et al., (2002, 2006); Mertin y Mohr, (2000); Moscicki, (1989) y Huerta, et al. (2012), que las víctimas que sufren maltrato presentan frecuentemente ansiedad y depresión, un nivel bajo de autoestima, así como un peor grado de adaptación a la vida cotidiana y un mayor riesgo de suicidio.

\section{CONCLUSIONES}

1. Las mujeres víctimas de violencia doméstica independientemente de su edad presentan estrés postraumático en sus tres componentes, re-experimentación, evitación y activación; alcanzando, en la escala a nivel global una media de 27.79 , dato alto con respecto al punto de corte que es 15 .

2. Como consecuencia del estrés postraumático, las mujeres víctimas de violencia doméstica presentan coeficientes mayores, en todas las edades, en el componente evitación con la inadaptación, es decir que cuando aumenta el TEPT aumenta la inadaptación por lo que no quiere aceptar ni tocar el tema, tendiendo a minimizar, ocultar o incluso a negar el maltrato del que son (o han sido) objeto, acrecentando el aislamiento de su familia y amigos.

3. Existe correlación significativa entre el aumento de la activación (componente del TEPT) con la inadaptación en todas la edades, cuando aumenta una de ellas también aumentará la otra pues la mujer víctima de violencia doméstica se encuentra en estado permanente de temor, causándoles respuestas inadaptativas, estado de ánimo irritable, problemas en su salud física y desesperanza. 
4. Todos los grupos de edades presentan síntomas de TEPT. Son las mujeres más jóvenes (16 a 21 años) quienes alcanzan una media relativamente más alta (29.67) en relación con los otros grupos de edades 22 a 35 años (27.81), 36 a 45 años (27.78 y 46 a 65 años (27.17) lo que hace más grave la permanencia en la relación de pareja y de mayor riesgo.

5. La edad es una variable independiente a los síntomas del estrés postraumático y a la inadaptación, no es la edad la que genera violencia, sino es la presencia de violencia en la relación de pareja en cualquier etapa de la vida, la que genera trastornos en la salud mental de la mujer víctima.

6. Los síntomas del trastorno de estrés postraumático son interdependientes con la inadaptación a la vida cotidiana, afectando la salud mental de la mujer víctima de violencia doméstica con permanente temor, aislamiento, desapego de los demás, y además obstaculiza y limita su vida afectiva, familiar, social y laboral (nivel de significancia .000).

7. La edad cronológica no es un factor de incidencia en la inadaptación de la mujer víctima de violencia doméstica, no se encontró ninguna razón F significativa. Observándose en todos los grupos de edades que las medias aritméticas son mayor de 2.5 indicador que, es la violencia doméstica independientemente de la edad la que afecta a la víctima en sus diferentes contextos y causa trastornos en su salud mental.

\section{REFERENCIAS BIBLIOGRÁFICAS}

Alonso, E. (2007). Mujeres víctimas de violencia domestica con trastorno de estrés post traumático: validación empírica de un programa de tratamiento. Tesis doctoral, Universidad Complutense. Madrid.

Amor, J. \& Bohórquez, I. (2004). Mujeres víctimas de maltrato doméstico. Facultad de Psicología, Universidad Nacional de Educación a Distancia, Madrid (España). Recuperado en www.fundacionfive.com/wp-content/uploads/Formacion13e.pdf

Arias, I., et al.. (1999). Prevalence and correlates of physical aggression during courtship. Journal of Interpersonal Violence, 2, 82-90.

Bardales, Olga (2006). Estado de las investigaciones sobre violencia familiar y sexual en el Perú 2001-2005. MIMDES. Lima

Blazquez, M. y Moreno, J. (2008). Maltrato psicológico en la pareja, Prevención y educación emocional. Editorial EOS. Madrid.

Calvete, Esther, et. al. (2007). Trastorno por estrés postraumático y su relación con esquemas cognitivos disfuncionales en mujeres maltratadas. En: Revista Psicothema 19 (3), 446-451. Asturias.

Cascardi, M., O'Leary, \& Schlee, K. A. (1999). Co-occurrence and Correlates of Posttraumatic Stress Disorder and Major Depression in Physically Abused Women. Journal of Family Violence, Vol. 14, $\mathrm{N}^{\circ} 3$, 199. New Jersey. USA.

Dutton, D. y Golant, S. (2004). El golpeador: un perfil psicológico. Paidós. Barcelona. (Segunda reimpresión). 
Echeburúa, Enrique, et.al. (2002). Mujeres maltratadas en convivencia prolongada con el agresor: variables relevantes. Rev. Acción Psicológica 2, 135-150. País Vasco. España

Echeburúa, Enrique, et. al. (2002). Repercusiones psicopatológicas de la violencia doméstica en la mujer en función de las circunstancias del maltrato. En: Revista Internacional de Psicología Clínica y de la Salud, 2 (2), 227-246. País Vasco. España.

Escartín, M. (2011). Violencia y mujer: la intervención de crisis con mujeres maltratadas y sus hijos. Boletín Informativo de Trabajo Social. No 16, España.

Domínguez, J., García, P., \& Cuberos, I. (2008). Violencia contra las mujeres en el ámbito doméstico: consecuencias sobre la salud psicosocial. En anales de Psicología, 24 (1), 115-120.

Golding, J. M. (1999). Intimate partner violence as a risk factor for mental disorders: A metaanalysis. Journal of Family Violence, vol. 14, 99-132.

Guezmes, Ana et. al. (2002). Violencia Sexual y Física contra las Mujeres en el Perú. Estudio Multicéntrico de la OMS sobre la violencia de pareja y la salud de las mujeres. Lima.

Informe de la Encuesta Demográfica y de Salud Familiar 2012. (2013) Instituto Nacional de Estadística. Lima

Informe de la Encuesta Demográfica y de Salud Familiar - ENDES 2004 (2006). Instituto Nacional de Estadística. Lima.

Labrador, Francisco, et. al. (2010). Características psicopatológicas de mujeres víctimas de violencia de pareja. En: Revista Psicothema 2010. Vol. 22, (1), 99-105. País Vasco, España.

Lorente, M. (2001). Mi marido me pega lo normal. Barcelona: Crítica.

MIMDES (2009). ¿Qué hacemos frente al feminicidio? Estadísticas y líneas de atención desde los Centros de Emergencia Mujer. CENDOC MIMDES. Lima.

Miljanovich, M. et. al. (2010). Perú: Mapa de violencia familiar, a nivel departamental, según la ENDES 2007-2008. Características e Implicancias. En: Revista de Investigación en Psicología. Vol. 13-2, 191-205. Lima.

Ministerio de la Mujer y Poblaciones vulnerables (2013). Compendio estadístico 2013 del MIMP. Recuperado en www.mimp.gob.pe/index.php?option =com_content\&view... id... Lima.

Sarasua, Belen, et. al. (2007). Perfil psicopatológico diferencial de las víctimas de violencia de pareja en función de la edad. En: Revista Psicothema 19 (3), 459-466. España.

Walker, L. (1999). Terapia para sobrevivientes con mujeres golpeadas. En: Revista Argentina de Clínica Psicológica. Vol. 8, 3, 201-210. Argentina. 